J. Clin. Chem. Clin. Biochem.

Vol. 20, 1982, pp. 305-312

\title{
Zur enzymatischen Analytik des HDL-Sphingomyelin
}

\author{
Von H. Schriewer, H.-U. Jabs und G. Assmann \\ Zentrallaboratorium der Medizinischen Einrichtungen der Westfälischen Wilhelms-Universität Münster \\ und Institut für Arterioskleroseforschung an der Westfälischen Wilhelms-Universität Münster
}

(Eingegangen am 28. Juli/25. November 1981)

Zusammenfassung: Es wird eine einfach durchzuführende enzymatische Bestimmungsmethode für Sphingomyelin im Apolipoprotein B-freien Überstand nach Fällung von Blutseren mit Phosphorwolframsäure/ $\mathrm{MgCl}_{2}$ (HDL-Sphingomyelin) beschrieben. Das Analysenprinzip beruht auf der durch Sphingomyelinase aus $B$. cereus katalysierten Spaltung von Sphingomyelin in Phosphorylcholin und N-Acylsphingosin und der anschließenden Freisetzung von Cholin aus Phosphorylcholin mittels alkalischer Phosphatase. Die Analytik von Cholin erfolgt mittels Cholinkinase im optischen Test.

Die mit der enzymatischen Methode ermittelten Sphingomyelinwerte stimmen gut mit den Werten überein, die mit der konventionellen chemischen Methode gemessen werden. Weiterhin findet man mit der enzymatischen Methode in der durch Ultrazentrifugation isolierten HDL-Fraktion $(1,063-1,21 \mathrm{~kg} / \mathrm{l})$ und der nach Fällung Apolipoprotein B-haltiger Lipoproteine isolierten Überstandsfraktion übereinstimmende Werte.

\section{The enzymatic analysis of sphingomyelin in $H D L$}

Summary: A simple method is described for the enzymatic determination of sphingomyelin in the apolipoprotein B-free supernatants prepared by precipitation of blood sera with phosphotungstate/ $\mathrm{MgCl}_{2}$. The analysis is based on the enzymatic hydrolysis of sphingomyelin, by sphingomyelinase from $B$. cereus, into phosphorylcholine and $\mathrm{N}$-acylsphingosine, and subsequent hydrolysis of phosphorylcholine by alkaline phosphatase. The choline formed is determined by choline kinase in an optical teșt.

The results from this method were in good agreement with those obtained by the conventional chemical sphingomyelin determination. Furthermore, there was a good correlation between the sphingomyelin concentrations obtained from the HDL fractions isolated by ultracentrifugation $(1.063-1.21 \mathrm{~kg} / \mathrm{l})$ and those obtained from the apolipoprotein $\mathrm{B}$ free supernatants af̣ter phosphotungstate/ $\mathbf{M g C} \mathbf{C l}_{2}$ precipitation of serạ.

\section{Einführüng}

Die Analytik der High Density-Lipoproteine (HDL) als Risikoindikator der koronaren Herzkrankheit hat in den letzten Jahren zunehmend an Bedeutung gewonnen $(1,2)$. Das bisher am breitęsten angewandte Verfahren der ,HDL-Quantifizierung " beruht auf der Bestimmung des Cholesterins im Präzipitationsüberstand nach Fällung Apolipoprotein B-haltiger Lipoproteine mit Polyanionen in Verbindung mit divalenten Kationen. Infolge der Heterogenität der HDL als Partikelgruppe mit unterschiedlicher Komposition, unterschiedlichem Metabolismus und unterschiedlichen physikochemischen Eigenschaften kann man jedoch vom HDL-Cholesterinwert nicht ohne weiteres auf die HDLMasse (und vice versa) schließen. HDL-Partikel bestehen etwa zu 50\% aus Proteinen, zu 25-30\% aus Phospho- lipiden, zu 10-20\% aus Cholesterin und Cholesterinestern und zu 3-5\% aus Triglyceriden. Obwohl der Phospholipidgehalt der HDL höher als der Cholesteringehalt dieser Partikel ist, hat die Bestimmung der HDLPhospholipide oder die Bestimmung der einzelnen Phospholipidfraktionen der HDL bisher wegen der aufwendigen Analytik keinen Eingang in die Routine gefunden.

Durch die Einführung kommerziell verfügbarer, spezifisch wirkenden Phospholipasen sowie der in letzter Zeit entwickelten empfindlichen Bestimmungsmethoden für Cholin ist es möglich, relativ rasch und einfach verschiedene cholinhaltige Phospholipidfraktionen zu analysieren. Uber die Analytik von Phosphatidylcholin im Fruchitwasser $(3,4)$ oder der gesamten cholinhal tigen Phospholipide im Blutserum (5) mit enzymatischen Bestimmungsverfahren liegen bereits Erfahrungen vor. 
Eine enzymatische Analytik der Subfraktionen des Phospholipidanteils der HDL (Phosphatidylcholin, Sphingomyelin, Lysophosphatidylcholin, Phosphatidylethanolamin und andere) wurde bisher noch nicht durchgeführt. Die bisherigen epidemiologischen und klinischen Ergebnisse bezüglich der Analytik von HDL-Cholesterin und HDL-Apolipoproteinen $(6,7)$ lassen vermuten, daß auch die differenzierte Phospholipidanalytik dieser Partikel die Frühdiagnostik und Prognostik der koronaren Herzkrankheit verbessern sollte. Wir möchten im folgenden über ein vollenzymatisches Sphingomyelin-Bestimmungsverfahren berichten, das wir zur Analytik von Sphingomyelin im Präzipitationsüberstand nach Fällung Apolipoprotein B-haltiger. Lipoproteine mit Phosphorwolframsäure $/ \mathrm{MgCl}_{2}$ ("HDL-Sphingomyelin") entwickelt haben.

\section{Material und Methoden}

\section{Substanzen}

(Methyl- ${ }^{14} \mathrm{C}$ ) Cholin spezifische Aktivität $2,2 \mathrm{GBq} / \mathrm{mmol}$, Amersham Buchler, Braunschweig

Phosphorwolframsäure $/ \mathrm{MgCl}_{2}$ Fällungsreagenz, Boehringer Mannheim, Test Nr. 400971

Sphingomyelinase aus $B$. cereus (Sphingomyelin choline-phosphohydrolase, EC 3.1.4.12), Boehringer Mannheim, Bestell-Nr. 396753

Alkalische Phosphatase, spezifische Aktivität $65 \mathrm{U} / \mathrm{mg}$ (Orthophosphoric-monoester phosphohydrolase (alkaline optimum) EC 3.1.3.1), Boehringer Mannheim, Bestell-Nr. 108162

Cholinkinase aus Hefe, spezifische Aktivität 0,5 U/mg (ATP: choline-phosphotransferase, EC 2.7.1.32), Boehringer Mannheim, Bestell-Nr. 348651

Lactatdehy drogenase, spezifische Aktivität $550 \mathrm{U} / \mathrm{mg}$ bei $25^{\circ} \mathrm{C}$ aus Kaninchenmuskel ( $L$-Lactate: NAD oxidoreductase, EC 1.1.1.27) Boehringer Mannheim, Bestell-Nr. 127330

Pyruvatkinase, spezifische Aktivität $200 \mathrm{U} / \mathrm{mg}$ bei $25^{\circ} \mathrm{C}$ aus Kaninchenmuskel (ATP: pyruvate 2-O-phosphotransferase, EC 2.7.1.40), Boehringer Mannheim, Bestell-Nr. 127418

\section{Probenmaterial}

Als Versuchsmaterial wurden Seren aus der Untersuchungsreihe „Prospektive epidemiologische Studie bei Betriebsangehörigen im Raum Westfalen verwandt. Die Blutabnahme erfolgte morgens nüchtern in einem speziell hierfür eingerichteten Omnibus. Das gewonnene Serum wurde bei $+4^{\circ} \mathrm{C}$ aufbewahrt und traf spätestens 3 Tage nach der Blutabnahme in unserem Laboratorium ein. Die Analytik erfolgte innerhalb von 24 Stunden nach Erhalt der Serumproben.

\section{HDL-Isolierung durch Ultrazentrifugation}

Die Ultrazentrifugation wurde im 40.3-Rotor (Beckman Instruments) durchgeführt. $3 \mathrm{ml}$ der Serumproben wurden mit einer $0,15 \mathrm{~mol} / 1 \mathrm{NaCl}$ enthaltenden Lösung auf $5 \mathrm{ml}$ verdünnt, mit festem Kaliumbromid auf eine Dichte von $1,063 \mathrm{~kg} / 1$ eingestellt, mit einer $1,063 \mathrm{~kg} / 1$ Kaliumbromid enthaltenden Lösung überschichtet und 24 Stunden bei $+4^{\circ} \mathrm{C}$ bei $36000 \mathrm{~min}^{-1}$ zentrifugiert. Die durch ,Schneiden“" der Röhrchen gewonnenen Fraktionen der Dichte $>1,063 \mathrm{~kg} / 1$ wurden mit einer $1,063 \mathrm{~kg} / 1$ $\mathrm{KBr}$-nthaltenden Lösung auf $5 \mathrm{ml}$ verdünnt, mit festem Kaliumbromid auf eine Dichte von $1,21 \mathrm{~kg} / 1$ eingestellt, mit einer 1,21 $\mathrm{kg} / \mathrm{l} \mathrm{KBr}$ enthaltenden Lösung überschichtet und 48 Stunden bei $36000 \mathrm{~min}^{-1}$ zentrifugiert. Die HDL-Fraktionen 1;063-1,21 $\mathrm{kg} / \mathrm{l}$ wurden nach erschöpfender Dialyse gegen $0,15 \mathrm{~mol} / 1 \mathrm{NaCl}$ mit der genannten $\mathrm{NaCl}-\mathrm{Lösung}$ auf das Ursprungsvolumen von $3 \mathrm{ml}$ aufgefüllt und bei $4^{\circ} \mathrm{C}$ aufbewahrt.

\section{HDL-Isolierung durch Präzipitation Apolipoprotein B-haltiger Lipoproteine}

Die Präzipitation Apolipoprotein B-haltiger Lipoproteine erfolgte mit Phosphorwolframsäure/ $\mathrm{MgCl}_{2}$ mit der Testkombination Boehringer Mannheim Nr. 400971 (2): 1 ml Blutserum wurde mit 0,1 ml Fällungsreagenz versetzt (Phosphorwolframsäure-Konzentration $3,64 \mathrm{~g} / \mathrm{l}, \mathrm{MgCl}_{2}$-Konzentration $45,5 \mathrm{mmol} / \mathrm{l}$ ) und $10 \mathrm{~min}$ bei Raumtemperatur stehen gelassen. Anschließend wurde $30 \mathrm{~min}$ bei $4^{\circ} \mathrm{C}$ und etwa $1500 \mathrm{~g}$ zentrifugiert. Der klare Uberstand wurde zur enzymatischen Sphingomyelinbestimmung eingesetzt.

\section{Enzymatische Sphingomyelinbestimmung}

Die enzymatische Sphingomyelinbestimmung wurde, wenn nicht anders beschrieben, nach folgendem Prinzip durchgeführt: Sphingomyelin $\stackrel{\text { Sphingomyelinase }}{\longrightarrow} \begin{aligned} & \text { N-Acyl-sphingosin } \\ & + \text { Phosphorylcholin }\end{aligned}$

\begin{tabular}{|c|c|}
\hline Phosphorylcholin & $\stackrel{\text { alkalische Phosphatase }}{\longrightarrow}$ Cholin + Phosphat \\
\hline Cholin + ATP & Phosphorylcholin + ADP \\
\hline $\begin{array}{l}\text { ADP + Phospho- } \\
\text { enolpyruvat }\end{array}$ & Pyruvatkinase \\
\hline
\end{tabular}

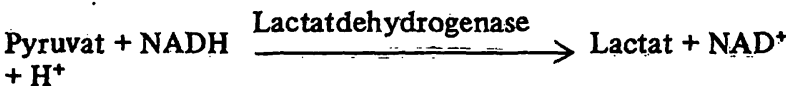

1. Enżymatische Spaltung vön Sphingom yelin

In Reaktionsgefäße (Sarstedt Nr. 52690) wurden pipettiert:

$0,5 \mathrm{ml}$ Glycinpuffer $(0,2 \mathrm{~mol} / 1$ Glycin, $10 \mathrm{mmol} / 1$

$\mathrm{MgSO}_{4} \times 7 \mathrm{H}_{2} \mathrm{O}, 0,1 \mathrm{~g} / 1$ Natriumdodecylsulfat, $\mathrm{pH} \mathrm{8,0)}$

$0,9 \mathrm{mi} 0,15 \mathrm{~mol} / 1 \mathrm{NaCl}$

$0,1 \mathrm{ml}$ Probe

$0,02 \mathrm{ml}$ Sphingomyelinase (2 $\mathrm{U}$ )

$0,01 \mathrm{ml}$ alkalische Phosphatase $(6,5 \mathrm{U})$.

Ein Reagenzienleerwert enthielt statt Probe ein entsprechendes Volumen $0,15 \mathrm{~mol} / 1 \mathrm{NaCl}$. Der Ansatz wurde 30 Minuten bei $37^{\circ} \mathrm{C}$ inkubiert, anschließend 10 Minuten im Thermoblock bei $95^{\circ} \mathrm{C}$ erhitzt und die denaturierten Proteine nạch Abkühlung der Lösung auf Raumtemperatur in einer Eppendorf-Zentrifuge 3200 abzentrifugiert.

\section{Enzymatische Messung des freigesetzten Cholins}

$1 \mathrm{ml}$ des erhaltenen Überstandes von Probenansatz bzw. Reagenzienleerwertansatz wurden in Eppendorf-K-Küvetten (Nr. 4071306.009) überführt,

$0,050 \mathrm{ml}$ Coenzymlösung ( $4 \mathrm{mmol} / 1 \mathrm{NADH}, 20 \mathrm{mmol} / 1 \mathrm{ATP}$, $7 \mathrm{mmol} / 1$ Phosphoenolpyruvat (kristallisiertes Tricyclohexylammoniumsalż), $45 \mathrm{mmol} / 1$ Glucose), $0,010 \mathrm{ml}$ Pyruvatkinase/ Lactatdehydrogenase-Gemisch (je $2300 \mathrm{kU} / \mathrm{l}$ ) hinzugefügt; der Reaktionsansatz gut gemischt und nach 10 Minuten Inkubation bei $37^{\circ} \mathrm{C}$ die Absorption $\mathrm{A}_{1}$ bei $365 \mathrm{~nm}$ gemessen (Messung gegen $\mathrm{H}_{2} \mathrm{O}$ ). Anschließend wurden

$0,025 \mathrm{ml}$ Cholinkinase $(2 \mathrm{kU} / \mathrm{l})$ hinzupipettiert, die Lösung gut gemischt und nach 30 Minuten Inkubation $A_{2}$, nach weiteren 30 Minuten $\mathbf{A}_{3}$ gemessen.

\section{Berechnung der Sphingom yelinkonzentration}

$\Delta A($ Probe, Leerwert $)=\left(A_{1}-\dot{A}_{2}\right)-\left(A_{2}-A_{3}\right)$

$\Delta \mathbf{A} \equiv \Delta \mathbf{A}_{\text {Probe }}=\Delta \mathbf{A}_{\text {Leerwert }}$

Konzentration $=\frac{\Delta \mathbf{A} \times 1,53 \times 1,085 \times 1,1}{3,44 \times 1 \times 0,1}[\mathrm{mmol} / \mathrm{l}]$

Bei der HDL-Isolierung mittels Ultrazentrifugation entfällt dex Verdünnungsfaktor 1,1 . $\rightarrow$ 


\section{Messung der Cholinkinasereaktion}

$0,1 \mathrm{ml}$ des nach Fällung Apolipoprotein B-haltiger Lipoproteine aus Serum mit Phosphorwolframsäure $/ \mathrm{MgCl}_{2}$ erhaltenen Úberstandes wurden mit $1 \mathrm{ml}$ Glycinpuffer (siehe oben) versetzt, 30 Minuten bei $37^{\circ} \mathrm{C}$ inkubiert und die Serumproteine $15 \mathrm{Mi}$ nuten bei $95^{\circ} \mathrm{C}$ denaturiert. Der Niederschlag wurde abzentrifugiert und zum Uberstand $0,185 \mathrm{mg}$ Cholin, [Methyl-14 $\mathrm{C}$ ]Cholin (etwa $400 \mathrm{Imp}$./min) und 0,1 U Cholinkinase gegeben. Nach 30 Minuten Inkubation bei $37^{\circ} \mathrm{C}$ wurde die Reaktion durch Erhitzen auf $95^{\circ} \mathrm{C}$ unterbrochen. Die Inkubation eines entsprechenden Blindwertes erfolgte ohne Anwesenheit von Cholinkinase.

$0.02 \mathrm{ml}$ des Reaktionsansatzes wurden auf Whatman $3 \mathrm{MM}$ Chromatographiepapier aufgetragen und das Chromatogramm im Laufmittel Isopropanol-Trichloressigsäure-0,27 kg/ $\mathrm{kg} \mathrm{NH}$ (Volumina, $75 \mathrm{ml}+25 \mathrm{ml}+0,3 \mathrm{ml}$ ) über $11 \mathrm{~cm}$ entwickelt. Nach dem Trocknen wurde das Chromatogramm in $0,5 \mathrm{~cm}$ breite Streifen zerschnitten, in Szintillationsröhrchen übergeführt und die Radioaktivität nach Zugabe von $1 \mathrm{ml} \mathrm{H}_{2} \mathrm{O}$ und $4 \mathrm{ml}$ Unisolve I (Zinsser, Frankfurt) im Szintillationszähler gezählt.

\section{Sphingomyelinbestimmung nach dünnschichtchromatographischer Trennung der Phospholipide}

\section{Phospholipidextraktionen}

Die Phospholipidextraktionen aus dem Überstand von Seren nach Fällung Apolipoprotein B-haltiger Lipoproteine mit Phosphorwolframsäure $/ \mathrm{MgCl}_{2}$ erfolgte nach Sundler et al. (8). $1 \mathrm{mi}$ Probe wurde mit dem 4-fachen Volumen Chloroform-Methanol $(1+1)$ versetzt und intensiv geschüttelt. Nach 12 Stunden wurden die denaturierten Proteine abzentrifugiert und der Überstand abgetrennt. Der Überstand wurde nochmals extrahiert, die Unterstände

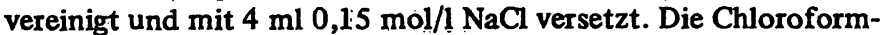
phase wurde durch Zentrifugation abgetrennt, im $\mathrm{N}_{2}$-Strom zur Trockne gebracht und in $0,050 \mathrm{ml}$ Chloroform aufgenommen. Pro Einzelanalyse wurden hiervon 0,005 $\mathrm{ml}$ (entsprechend 10-20 $\mu \mathrm{g}$ Sphingomyelin) dünnnschịchtchromatographisch aufgetrennt.

\section{Dünnschichtchriomatographie der Phospholipide}

Die analytische Trennung von Phosphölipiden wurde auf HPTLC-Platten mit Konzentrierungszone (Kieselgel 60, Merck Darmstadt, Nr. $13748,10 \times 10 \mathrm{~cm}$ ) durchgefuihrt. Als Laufmittel diente Chloroform-Methanol-Wasser (Voluminina, $60 \mathrm{ml}+25 \mathrm{ml}$ $+4 \mathrm{ml}$ ) bei Kammersättigung. Zur Darstellung der getrennten Fraktionen wurde die Platte mit einer $100 \mathrm{~g} / \mathrm{i}$ ethanolischen Molybdatophosphorsäurelösung besprüht, bei $140^{\circ} \mathrm{C} 15$ Minuten erhitzt und die nicht mit dem Reagenz blau gefärbten Zonen mit Amminoniàkdämpfen entfärbt.

Zur präparativen Abtrennung Von Sphingomyelin wurden die getrennten Fraktionen in einem geschlossenen Gefäß mit Iod eingefärbt, markiert und die sphingomyelinhaltigen Positionen nach Abkratzen mit einer Rasierklinge in Zentrifugierröhrchen überführt. Die Elution aus dem Kieselgel erfolgte nach Skipski (9).

\section{Phosphatbestimmung}

Die Phosphatbestimmung nach Hydrolyse der Phospholipide mït Perchlorsäure wurde nach Bartlett (10) durchgeftihitit. Die Kalibrierung erfolgte durch eine aus Analysen verschiedener $\mathrm{KH}_{2} \mathrm{PO}_{4}$-Lösungen erstellten Standardkurve.

\section{Ergebnisse}

\section{Optimale Sphingomyelinaseaktivität}

Zur Uberprüfung der zur Sphingomyelinspaltung optimalen Bedingungen wurde der Reaktionșansatz nach Zusatz von Sphingomyelinase und Inkubation delipi- diert und nicht hydrolysiertes Sphingomyelin sowie N-Acyl-sphingosin als Reaktionsprodukt dünnschichtchromatographisch aus dem Lipidextrakt abgetrennt. Die dünnschichtchromatographische Auftrennung der Lipidextrakte von Inkubationsansätzen, die $1 \mathrm{U}$ Sphingomyelinase und etwa $200 \mu \mathrm{g}$ Sphingomyelin pro Reaktionsansatz enthielten, ließ kein ungespaltenes Sphingomyelin erkennen (untere Nachweisgrenze für Sphingomyelin $0,15 \mu \mathrm{g}$ ). Wurde zur Inkubation eine Sphingomyelinaseaktivität von $<0,5 \mathrm{U} /$ Reaktionsansatz eingesetzt, ließ sich ungespaltenes Sphingomyelin dünnschichtchromatographisch nachweisen.

\section{pH-Abhängigkeit der Sphingomyelinspaltung}

Sphingomyelin wurde bei einem pH-Wert von $\leq 6,4$ und in Gegenwart von $1 \mathrm{U}$ Sphingomyelinase im Reaktionsansatz vollständig hydrolysiert (pH-Optimum 5,9-6,4). Lag der pH-Wert im Inkubationsansatz unter den gewählten Reaktionsbedingungen bei $>7,0$, zeigte die dünnschichtchromatographische Auftrennung der entsprechenden Lipidextrakte ungespaltenes Sphingomyelin.

Ohne das Detergens Natriumdodecylsulfat erfolgte unter den gewählten Inkubationsbedingungen nur eine unvollständige Sphingomyelinhydrolyse. Die Dünnschichtchromatographie des entsprechenden Lipidextraktes ergab einen noch positiven Sphingomyelinnachweis. Wurde die Inkubation unter sonst gleichen Bedingungen in Anwesenheit von $0,1 \mathrm{~g} / \mathrm{l}$ Natriumdodecylsulfat durchgeführt, erfolgte eine vollständige Sphingomyelinhydrolyse. Detergenskonzentrationen $\geq 0,5 \mathrm{~g} / 1$ bewirkten eine Hemmung der Sphingomyelinhydrolyse.

\section{Enzymatische Cholinbestimmung}

\section{Reaktionsverlauf}

Der Reaktionsverlauf der enżymatischen Cholinbestimmung ist in Abbildung 1 dargestellt. Nach Mischen von Probe bzw. 0,15 mol/1 NaCl (Reagenzienleerwert) mit den Coenzymen und Pyruvatkinase/Lactatdehydro-

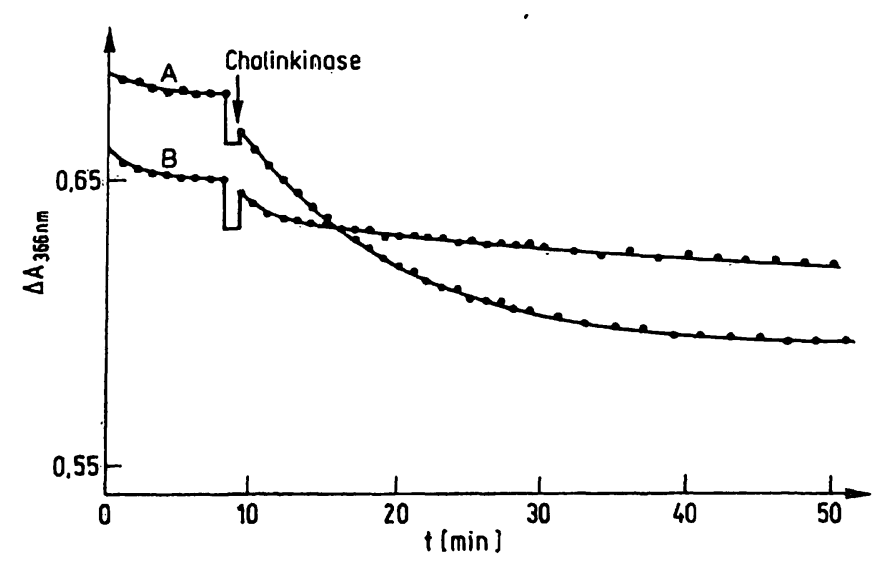

Abb. 1. Reaktionsverlauf der enzymatischen Cholinbestimmung: Reaktionsverlauf des Probenansatzes (A) und Reagentienleerwertansatzes $(B)(0,15 \mathrm{~mol} / 1 \mathrm{NaCl})$. 
genase beobachtet man eine „Vorreaktion", die nach etwa 10 Minuten Inkubationszeit beendet ist. Die nach Zugabe von Cholinkinase ausgelöste Absorptionsabnahme ist nach etwa 20 Minuten beendet. Der nach Ablauf der Reaktion weiter zu beobachtende ,Schleich“" (weitere gleichmäßige Absorptionsabnahme) war beim Probenansatz und Reagentienleerwertansatz unterschiedlich; daher ist eine dritte Absorptionsmessung $\left(\mathrm{A}_{3}\right)$ notwendig.

\section{Reaktionsbedingungen der enzymatischen Cholin- bestimmung}

Die Bedingungen der Umwandlung von Cholin in Phosphorylcholin wurden durch Zugabe von [Methyl- ${ }^{14} \mathrm{C}$ ]Cholin zum denaturierten, nach Sphingomyelinspaltung erhaltenen Zentrifugationsüberstand überprifit. Die danach im Testansatz vorhandene Cholinkonzentration betrug etwa das Tausendfache der normalerweise im Sphingomyelinansatz freigesetzten Cholinkonzentration, so daß die Umwandlung von $\left[{ }^{14} \mathrm{C}\right]$ Cholin in
Phosphoryl $\left.{ }^{14} \mathrm{C}\right]$ Cholin nicht quantitativ erfolgen konnte. Ein Maß für die Umwandlung war die Radioaktivitätsverteilung nach papierchromatographischer Auftrennung des Inkubạtionsansatzes. Wie Abbildung 2 zeigt, lag das pH-Optimum der Cholinkinase bei pH 8-9. Wir fanden nach Aufarbeitung der bei diesen $\mathrm{pH}$. Werten durchgeführten Reaktionsansätze nur einen relativen Anteil von 0,13-0,15 der Gesamtradioaktivität in der $\left[{ }^{14} \mathrm{C}\right.$-Methyl] Cholin-Fraktion, bei Aufärbeitung des bei pH 6 durchgeführten Reaktionsansatzes dagegen einen relativen Anteil von etwà 0,45 an der Gesamtradioaktivität.

Zur Überprüfung der Detergentienäbhängigkeit der Cholinkinase wurde die Koñzentrătión von Natriumdọdecylsulfat zwischen $0,1 \mathrm{~g} / \mathrm{l}$ und $5 \mathrm{~g} / \mathrm{l}$ eingestellt. Ohne $\mathrm{Nà}$ triumdodecylsulfat befand sich ein relativer Anteil . von 0,45 der Gesamtradioaktivität in der Phosphoryl$\left[{ }^{14} \mathrm{C}\right]$ Cholin-Fraktion, während bei Inkubation mit 0,1 $\mathrm{g} / 1$ Natriumdodecylsulfat ein relativer Anteil von $0 ; 60$ und bei Inkuibation mít $1 \mathrm{~g} / \mathrm{l}$ Natriumdodecylsulfat ein relativer Anteil von 0,65 der Gesamintradioaktivität in

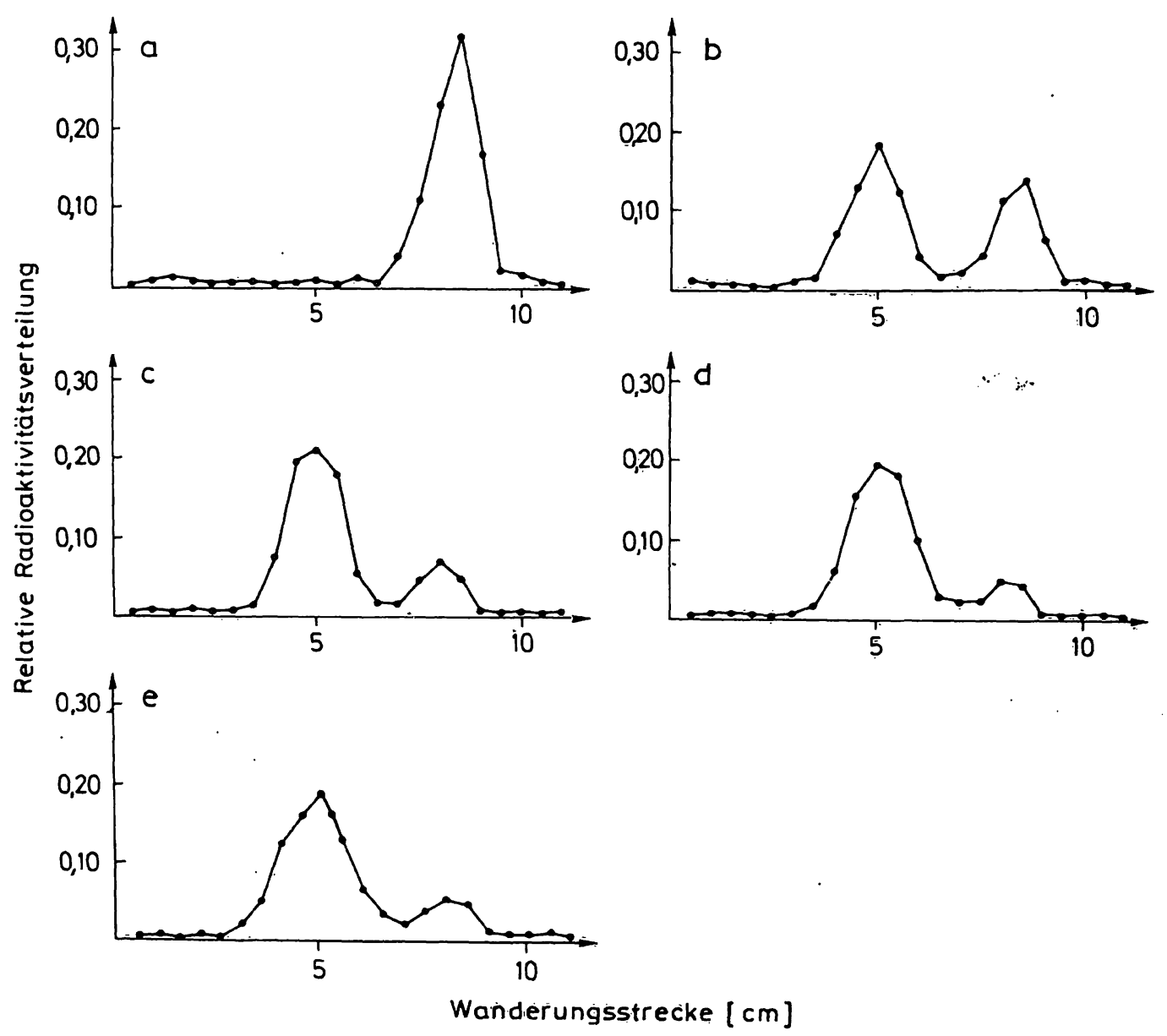

Abb. 2. pH-Abhängigkeit der Cholinkinasereaktion. Relative Radioaktivitätsverteilung von Phosphoryl[ $\left.{ }^{14} \mathrm{C}\right] \mathrm{Cholin}(1$. Peak) und $\left[{ }^{14} \mathrm{C}\right]$ Cholin (2. Peak) nach papierchromatographischer Auftrennung dès Inkubationsansatzes.

$\mathrm{a}=$ Inkubation in Abwesenheit von Cholinkinase;

$\mathrm{b}=$ Inkubation bei pH 6,0;

$\mathrm{c}=$ Inkubation bei pH 7,0;

$\mathrm{d}=$ Inkubation bei pH 8,0;

$\mathrm{e}=$ Inkubation bei pH 9,0 . 
dieser Fraktion gefunden wurde (Abb. 3). Bei höheren Konzentrationen von Natriumdodecylsulfat $(5-10 \mathrm{~g} / \mathrm{l})$ wurde die Cholinkinasereaktion vollständig gehemmt.

\section{Linearität der Bestimmungsmethode}

Die gemessenen Absorptionsdifferenzen waren in dem geprüften Bereich zwischen 20 bis $100 \mu$ l Probe (Apolipoprotein B-freier Fällungsüberstand) pro Inkubationsansatz den zugegebenen Probenvolumina direkt proportional (Abb. 4). Bei Zugabe von mehr als $100 \mu \mathrm{l}$ Probe zum Inkubationsansatz kam es im Verlauf der Sphingomyelinspaltungsreaktion zu Trübungen, die sich durch Zentrifugation nicht beseitigen ließen.

Die Linearität der Cholinbestimmung wurde durch Zugabe verschiedener Mengen eines eingewogenen
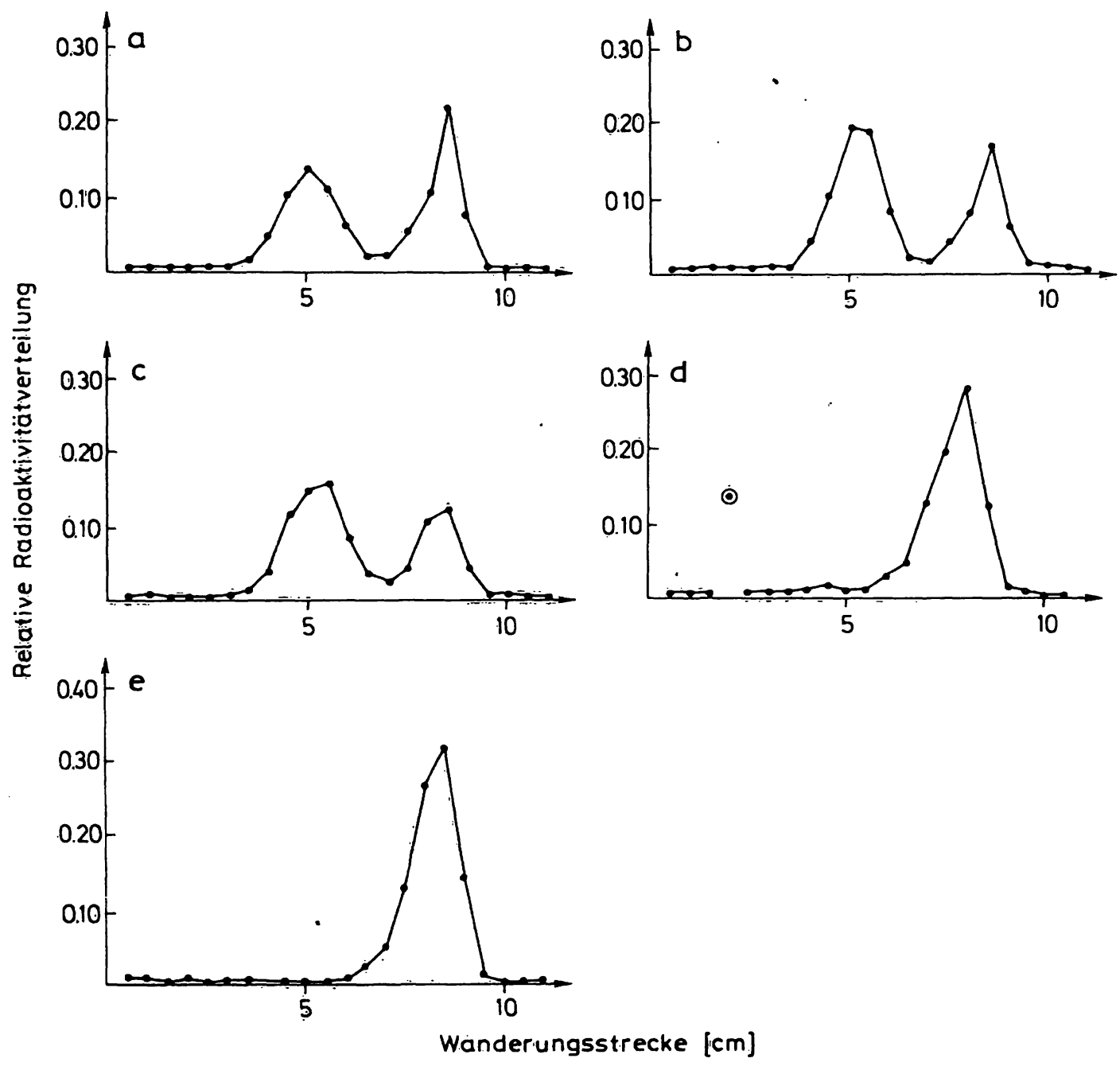

Abb. 3. Abhängigkeit der Cholinkînasereaktion von der Natriumdodecylsulfatkonzentration im Inkubationsansatz. Relative Radioaktivitätsverteilung von Phosphoryl[ $\left.{ }^{14} \mathrm{C}\right]$ Cholin (1. Peak) und $\left[{ }^{14} \mathrm{C}\right] \mathrm{Cholin}$ (2. Peak) nach papierchromatographischer Auftrennung des Inkubationsansatzes.

$a=$ Inkubation ohne Zusatz von Natriumdodecylsulfat;

$\mathrm{b}=$ Inkubation in Anwesenheit von $0,1 \mathrm{~g} / 1$ Natriumdodecylsulfat;

$c=$ Inkubation in Anwesenheit von $1 \mathrm{~g} / 1$ Natriumdodecylsulfat;

$\mathrm{d}=$ Inkubation in Anwesenheit von $5 \mathrm{~g} / \mathrm{l}$ Natriumdodecylsulfat;

$\mathrm{e}=$ Inkubation in Anwesenheit von $10 \mathrm{~g} / 1$ Natriumdodecylsulfat.

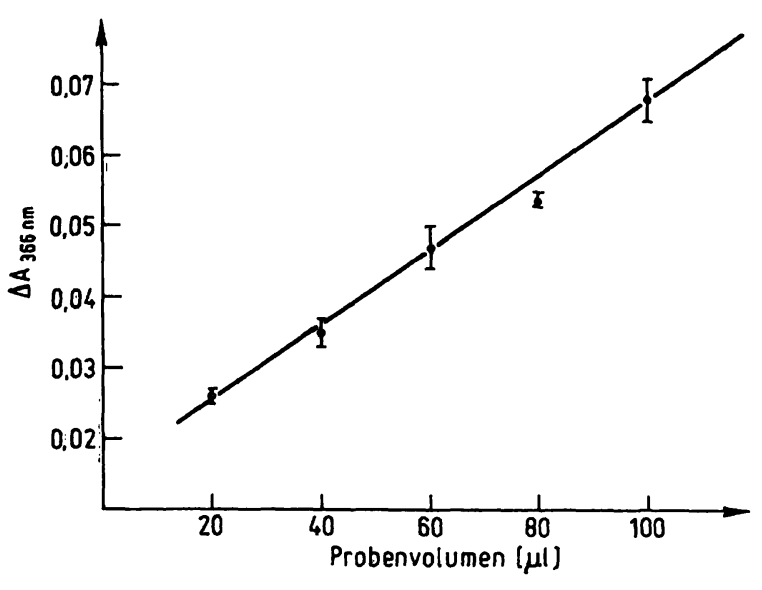

Abb. 4. Abhängigkeit der enzymatischen Cholinbestimmung von dem zum Sphingomyelinbestimmungsansatz zugegebenen Volumen an Apolipoprotein B-freiem Fällungsüberstand von Seren (Mittelwert \pm Standardabweichung von jeweils

10 4 Ansätzen). 
Cholinstandards zum Apolipoprotein B-freien Fällungsüberstand überprüft (Abb. 5). In dem geprüften Bereich war die Wiederfindungsrate zwischen 0,17 und 0,5 $\mu \mathrm{mol}$ Cholin pro Bestimmungsansatz der zugegebenen Cholinmenge proportional. Im Bereich über $0,5 \mu \mathrm{mol}$ an zugesetztem Cholin ermittelten wir nur einen relativen Anteil von 0,6-0,7 der theoretischen Cholinkonzentration. Offenbar reichen die im Testansatz vorliegenden Reagentienkonzentrationen bei Cholinkonzentrationen $>0,5 \mu \mathrm{mol}$ pro Testansatz nicht mehr für einen vollständigen Reaktionsablauf aus. Es ist jedoch zu berücksichtigen, daß bei Spaltung von HDL-Sphingomyelin die im Testansatz vorliegende Cholinmenge nur etwa $0,02 \mu \mathrm{mol}$ beträgt.

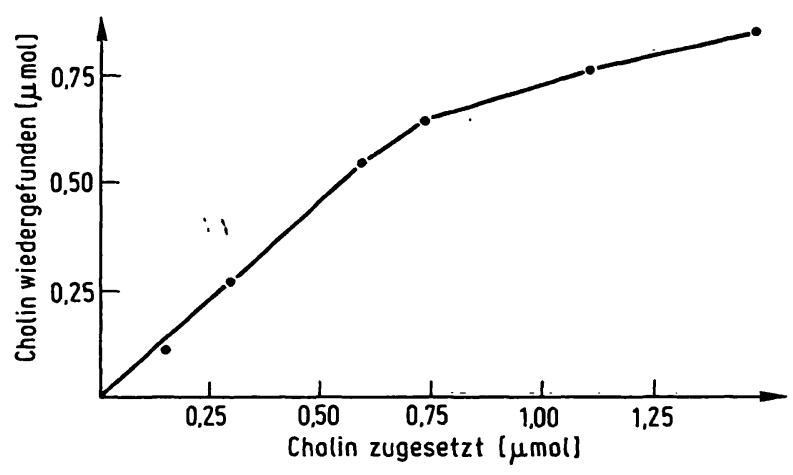

Abb. 5. Linearität der enzymatischen Cholinbestimmung. Abhängigkeit der Cholinwiederfindung von der zum Apolipoprotein B-freiem Fällungsüberstand zugegebenen Cholinmenge.

Vergleich der Sphingomyelinkonzentration in unterschiedlich isolierten HDL-Fráktionen

Die Regressionsanalyse der Meßdaten aus dem Apolipoprotein B-freien Präzipitationsüberstand und aus der durch Ultrazentrifugation isolierten HDL-Fraktion (1,063-1,21 kg/l) zeigte, daß die Sphingomyelinkonzentrationen im Apolipoprotein B-freien Fällungsüberstand mit den Sphingomyelinkonzentrationen in der durch Ultrazentrifugation isolierten HDL-Fraktion relativ gut übereinstimmen $(r=0,900, y=1,06 x-0,04$, $\mathrm{n}=32)($ Abb. 6).

\section{Rich tigkeit der Sphingomy elinbestim mung}

Die Richtigkeit der enzymatischen Sphingomyelinbestimmung wurde anhand der Sphingomyelinwerte überprüft, die mit der konventionellen Standardmethode (dünnschichtchromatographische Sphingomyelintrennung nach Lipidextraktion, Phosphatbestimmung nach Hydrolyse mit Perchlorsäure) ermittelt wurden. Da Vorversuche ergaben, daß die aus dem Apolipoprotein B-freien Fällungsüberstand isolierten Sphingomyelinfraktionen mit Phosphorwolframsäure kontaminiert waren, konnte die konventionelle Standardme thode nur bei HDL-Fraktionen eingesetzt

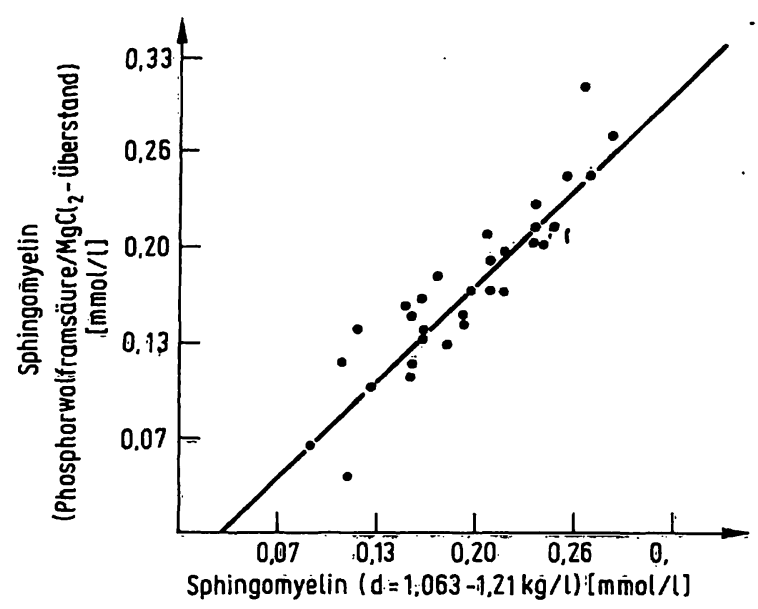

Abb. 6. Regressionsanalyse der Sphingomyelinwerte, die aụs der durch Ultrazentrifugation isolierten HDL-Fraktionen $(1,063-1,21 \mathrm{~kg} / 1)$ und der durch $\mathrm{F}$ ällung Apölipoprotein B-haltiger Lipoproteine isolierten Überstandsfraktionen ermittelt wurden; $y=1,06 x-0,04, r=0,900, n=32$.

werden, die durch sequentielle Ultrazentrifugation $(1,063-1,21 \mathrm{~kg} / 1)$ isoliert worden waren. Wie Abbildung 7 zeiğt, findet man bei Gegenüberstellung der mit der enzymatischen Sphingomyelinbestimmung und der konventionellen chemischen Methode ermittelten Werte eine gute Ubereinstimmung $(r=0,94, y=0,867 x+$ $0,02, n=20$ ).

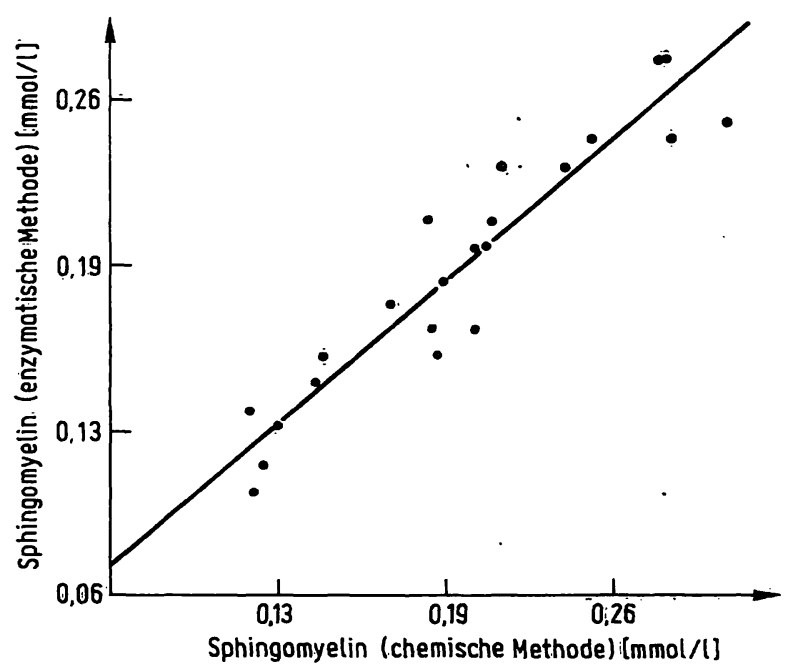

Abb. 7. Regressionsanalyse der Sphingomyelinwertè die mit der chemischen Methode und der enzymatischen Methode analysiert wurden; $y=0,867 x+0,02, x=0,94, n=20$.

\section{Präzision der Sphingomyelinbestim mung}

Wir fanden bezügliç der Präzisision in der Sèrie einen Variationskoeffizienten von $5,7 \%(\bar{x}=0,241 \mathrm{mmol} / \mathrm{l}$, $\mathrm{n}=21$ ).

Die Präzision von Tag zu Tag wurde von uns mit Serumproben ermittelt, die aus einem frischen Poolserum in 
Aliquoten abgefullt und bei $-20^{\circ} \mathrm{C}$ gelagert wurden. In Vorversuchen wurde ermittelt, daß die bei $-20^{\circ} \mathrm{C}$ tiefgefrorenen Seren über den von uns geprüften Zeitraum von 4 Wochen keine Unterschiede der Sphingomyelinwerte im Apolipoprotein B-freien Uberstand im Vergleich zu frischen Seren aufwiesen. Der aus diesen Proben ermittelte Variationskoeffizient von Tag zu Tag betrug 5,3\% $(\mathrm{x}=0,195 \mathrm{mmol} / \mathrm{l}, \mathrm{n}=12)$.

\section{Diskussion}

Im Rahmen der Verfügbarkeit spezifisch spaltender Phospholipasen sind in letzter Zeit verschiedene Methoden zur Quantifizierung von cholinhaltigen Phospholipiden im Fruchtwasser und im Blutserum entwickelt worden $(3,4,5,11,12)$. Zur Hydrolyse der cholinhaltigen Phospholipide wird entweder Phospholipase D aus Streptomyces chromofuscus, Phospholipase $C$ aus $B$. cereus oder Sphingomyelinase aus $B$. cereus eingesetzt und Cholin aus dem Hydrolyseprodukt Phosphorylcholin mittels alkalischer Phosphatase freigesetzt. Die Analytik des Cholins erfolgt entweder mittels Cholinkinase im optischen Test (3) bzw. bei Verwendung von $\left[\gamma_{-}{ }^{32} \mathrm{P}\right]$ ATP durch Abtrennung von $\left[\gamma_{-}{ }^{32} \mathrm{P}\right] \mathrm{Phos}-$ phorylcholin mittels Anionenaustauscherchromatographie (4) oder kolorimetrisch mittels Cholinoxidase und Peroxidase $(5,12)$. Die von uns zur Sphingomyelinbestimmung im Apolipoprotein B-freien Überstand nach Fällung von Seren mit Phosphorwolframsäure/ $\mathrm{MgCl}_{2}$ entwickelte Methode basiert auf der Verwendung von Sphingomyelinase und Cholinkinase. Die erzielten Analysenergebnisse stimmen gut mit den Sphingomyelinwerten übèrein, die durch die konventionelle Standardmethode ermittelt wurden (dünnschichtchromatographische Sphingomyelintrennung, Phosphàtbestimmung nach Hydrolyse mit Perchlorsäure). Die Konzentration des dem Testanșąz zugesetżten Detergens Natriumdodecylsulfat wurde von uns so gewählt, daß einerseits eine vollständige Sphingomyelinspaltung gewährleistet war und andererseits eine Hemmung der Cholinkinase vermieden wurde.

Die vorliegenden Ergebnisse żeigen, daß der Sphingomyelingehalt im Fällungsïberstand nạch Präzipitation Apolipoprotein B-haltiger Lipoproteine gut mit dem Sphingomyelingehalt in der durch Ultrazentrifugation getrennten HDL-Fraktion übereinstimmt. Dies läßt därauf schließen, daß während der Fällung Apolipoprotein B-haltiger Lipoproteine keine wesentliche Änderung im Sphingomyelingehalt der HDL auftritt bzw. der Sphingomyelinanteil der HDL-Partikel nicht mitpräzipitiert wird. Die erzielten Ergebnisse zeigen weiterhin, daß in dem geprüften Bereich zwischen
20-100 $\mu$ Probe/Bestimmungsansatz (Fällungsüberstand nach Fällung Apolipoprotein B-haltiger Lipoproteine mit Phosphorwolframsäure $/ \mathrm{MgCl}_{2}$ ) keine Beeinflussung der Bestimmungsmethode durch die Fällungsreagenzien auftritt.

Die ermittelten Variationskoeffizienten für die Präzision in der Serie und von Tag zu Tag liegen mit etwa $6 \%$ durchaus in einem akzeptablen Bereich. Eine wesentliche Erhöhung der Präzision ist zur Zeit nicht möglich, da die kommerziell verfügbare Cholinkinase nicht $\mathrm{zu}$ beseitigende Nebenaktivitäten an Hexokinase enthält (13). In Glucose-haltigen Proben wie Serum oder Serumfraktionen (Apolipoprotein B-freier Fällungsüberstand) kommt es deshalb zu einer unspezifischen NADH-Konzentrationsabnahme (,Schleich"). Wir fanden mit verschiedenen Chargen Cholinkinase unspezifische Absorptionsabnahmen zwischen 0,02-0,06/10 min Inkubation. Der durch die Hexokinase-Reaktion hervorgerufene "Schleich“ wird zwar durch den Abzug der Absorptionsdifferenz $A_{2}-A_{3}$ korregiert. Es hat sich jedoch bei der Überprüfung von Präzision und Richtigkeit als sinnvoll.erwiesen, den zwischen Test und Reagentienleerwertansatz bestehenden „Schleich-Unterschied“ durch Zugabe einer der Substratsättigung der Hexokinase entsprechenden Glucosekonzentration zum Cholinbestimmungsansatz auszugleichen.

Die von uns entwickelte enzymatische Bestimmungsmethode für HDL-Sphingomyelin ist trotz der relativ hohen Analysendauer erheblich schneller und weniger aufwendig als das bisherige konventionelle Bestimmungsverfahren. Die Reagenzienkosten der enzymatischen Sphingomyelinanalyse liegen zur Zeit bei etwa DM 5,30 pro Test, bedingt durch die relativ teure Sphingomyelinâse. Die Analytik des freigesetzten Cholins läßt sich im Prinzip durch die Verwiendung von Cholinoxidase und Peroxidase anstatt von Cholinkinase vereinfachen. Die kolorimetrische Cholinbestimmung ist jedoch zur Zeit noch kostenungünstiger als die beschriebene Cholinbestimmung im optischēn Test. Angesichts der ermittelten Daten bežiglich Präzision und Richtigkeit ist der Einsatz der enzymatischen HDL-Sphingomyelin-Bestimmungsmethode trotz der zur Zeit noch relativ hohen Analysenkosten zumindest für Forschungszwecke zu empfehlen. Eine breite Anwendung des enzymatischen HDLSphingomyelinbestimmungsverfahrens in der Routine ist von der klinischen Relevanz zukünftiger Ergebnisse abhängig.

\section{Acknowledgement}

Supported by the Deutsche Forschungsgemeinschaft and the Bundesministerium für Forschung und Technologie, Bonn.

\section{Literatur}

1. Assmann, G. \& Schriewer, H. (1981) J. Clin. Chem. Clin. Biochem. 19, 1-6.
2. Assmann, G., Schriewer, H. \& Funke, H. (1981) J. Clin. Chem. Clin. Biochem. 19, 273-278. 
3. Diedrich, K., Hepp, S., Welker, H., Krebs, D., Beuttler, H.-O. \& Michal, G. (1979) Geburtsh. Frauenheilk. 39, $849-856$.

4. McDonald, L. J., Robin, N. I. \& Siegel, L. (1981) Clin. Chem. 27, 410-416.

5. Takayama, M., Itoh, S., Nagasaki, T. \& Tanimizu, I. (1977) Clin. Chim. Acta 79, 93-98.

6. Assmann, G., Schriewer, H. \& Oberwittier, W. (1980)

Klin. Wochenschr. 58, 757-765.

7. Assmann, G., Funke, H. \& Schriewer, H. (1982) J. Clin. Chem. Clin. Biochem. 20, 287-28y.
8. Sundler, R. \& Akesson, B. (1975) Biochem. J, 146; 309-315.

9. Skipski, V. P., Peterson, R. F. \& Barclay, M. (1964) Biochem. J. 90, 374-378.

10. Bartlett, G. R. (1959) J. Biol. Chem. 24, 466-468.

11. Quershi, M. J., Murphỳ, G. M. \& Dowling, R. H. (1980)

Clin. Chim. Acta 105, 407-410.

12. Gurantz, D., Laker, M. F. \& Hofmạn, A.'F. (1981) J. Lipid. Res. 22, 373-376.

13. Ziegenhorn, J., persönliche Mitteilung.

Prof. Dr. G. Assmann

Zentrallaboratorịum der Meḍ. Eiñorichtựngen Westfälische Wilhelms-Universität

Westring 3

D-4400 Münster 